\title{
The effects of machining settings on the behavior of pulse trains in the WEDM process
}

\author{
Y.S. Liao*, J.C. Woo \\ Department of Mechanical Engincering, Vanional Tainan Enitersity. No. i Rossetelt Road, Sectim 4. Taipei 10617. Tainan
}

Received 12 April 1996

\begin{abstract}
A pulse discrimination system has been developed to study the characteristics of pulse trains in the WEDM process under various machining conditions. The effects of on-time, off-time and feed on the variations of the proportions of short sparks. arc sparks, and normal sparks in the total sparks (defined as short ratio, arc ratio, and normal ratio, respectively); the distribution of the ignition delay time of the normal sparks; the average ignition delay time; and the proportion of machining time in a specific sampling period were investigated. An approximate method for estimating the variation of the average gap width is also introduced. It is found that a short on-time, or a long off-time, or a large feed, results in the decrease of both normal ratio and average ignition delay time, and an increase of short ratio. On-time has the largest effect on the arc ratio, whilst off-time has the least effect. The ignition delay time of the normal sparks displays a reverse $\mathbf{J}$-shape distribution. On-time is the most prominent factor amongst all of the machining settings under consideration atfecting this distribution. In addition, the distribution becomes more left skew with a higher peak and a shorter tail under the condition of a shorter on-time setting. Based on the analysis results, the instability during the WEDM process can be attributed to an apparent increase of either arc sparks, short sparks, or both. A strategy to improve machining stability with respest to each case is proposed accordingly. 1997 Elsevier Science S.A.
\end{abstract}

Kelwords: WEDM process: Pulse trains: Machine settings

\section{Introduction}

The exact mechanism of the wire electro-discharge machining (WEDM) process is not yet clearly understood, because it is difficult to comprehend machining phenomena exactly due to multi-parameter machining settings (off-time, on-time, table feed, wire feed, etc.) and workpiece properties (materials, thickness). Experimental studies using a single-spark lead to valuable results, but these studies do not faithfully reflect the actual process where sparks occur at high frequency $(10-100 \mathrm{KHz})$. Works on statistical aspects of pulse trains indicate that successive effects are important both with breakdown cnaracteristics, and the distribution of slags in the gap [1]. Snoeys et al. [2,3] classified discharge pulses in die sinking EDM into several categories by two voltage threshold values obtained through many investigations, which provides an impor-

*Corresponding author. Tel.: +88623630231. tant approach to evaluate discharge condition quantitatively. However, it is difficult to apply in a high speed and high peak current pulse generator due to violent electromagnetic interference (EMI) and high power discharge oscillation. Watanabe et al. [4] classified discharge pulses into batches. They discriminated arc and normal spark by a threshold level which was based on the spark current peak and the spark voltage histogram and its distribution characteristics. This paper also contributes to the quantitative assessment of machining conditions and off-line process optimization. However, the special feature in this discrimination is that the threshold level changes with machining settings. Further, its value depends on the machining settings. In recent yeats, many researchers have investigated the analysis of pulse trains [5-8], their studies revealing that pulse trains can respond sensitively to the variations of the process. Accordingly, approaches to improve the performance of wire electro-discharge machines by the use of pulse train characteristics were studied [9-11]. Rajurkar and Wang proposed a strategy 
to control the sparking frequency so as to prevent wire from breaking according to the on-line identified workpiece height when cutting a workpiece with variable height [9]. The symptoms reflected in the variations of pulse trains during the wire rupture process have been identified in the present authors' previous investigation [10]. The abnormal ratio (the proportion of the arc and short spark in a specific time interval) and the sparking frequency were used as controlled parameters, an optimum and high-speed machining state being achieved without the risk of rupture of the wire [11].

In this present paper, the relationships between the machining settings and the pulse trains are analyzed by a pulse train discriminating system developed in the authors' laboratory. The behaviour of pulse trains under various machining setti.igs are analyzed quantitatively in terms of short ratio $R_{\mathrm{s}}$, arc ratio $R_{\mathrm{a}}$, normal ratio $R_{\mathrm{n}}$ (defined as the proportion of a particular spark in a specific time interval), machining time ratio $\left(t_{\mathrm{a}} / t_{\mathrm{t}}\right.$, total actual machining time in a specific time interval), distribution of ignition delay time and average ignition delay time of normal sparks. The variation of the distribution of ignition delay time of normal sparks is presented graphically. The results are discussed and explained.

\section{Pulse train discriminating system}

The power supply circuit of the wire cutter used in the experiment is shown in Fig. 1. This circuit has the characteristics of applying low power to lead the gap to discharge, followed by high power discharging which contributes to metal removal. The timing chart of pulse control signal is given in Fig. 2. The pulse control is accomplished by the following steps. The low power is turned on first by supplying $110 \mathrm{~V}$ voltage which causes

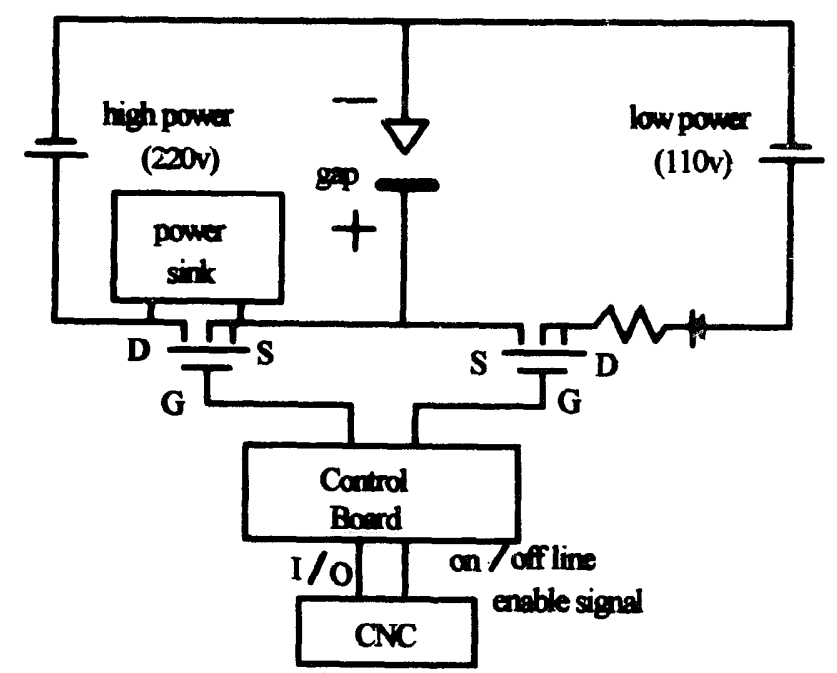

Fig. 1. WEDM power supply circuit.

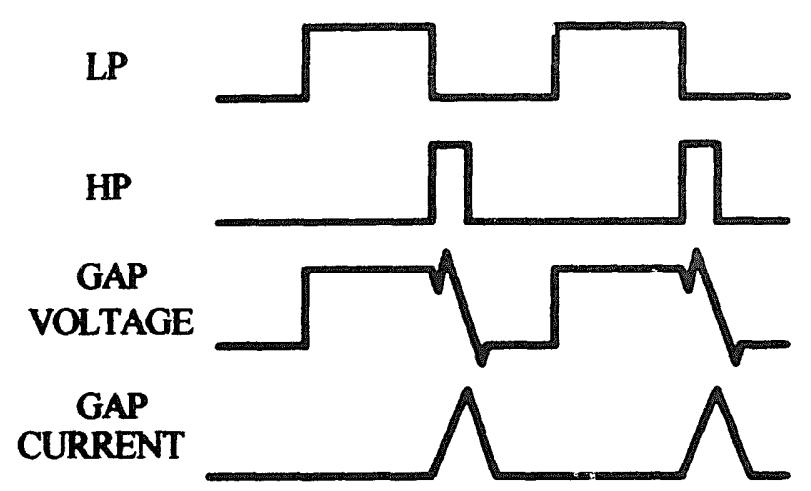

Fig. 2. Timing chart of the pulse control signal and the associated pulse trains of WEDM.

the gap to discharge. In the second stage, the low power is turned off whilst the high power of $220 \mathrm{~V}$ is turned on. After a short period of time $(0.5 \mu \mathrm{s})$ the gap is discharged with a high current. The gap remains in an insulated state until the low power is turned on again, and the next cycle starts. Typical gap voltage and the associated current waveforms are shown in Fig. 3. There are three sparks shown in the figure, namely short, arc, and normal sparks.

In order that various sparks can be differentiated, a knowledge of their characteristics is needed. When metal contact between the workpiece and the electrode takes place, a short spark occurs. At this moment, a short current occurs whilst the gap voltage is loaded synchronously, hence, there is no time lag between them. Arc spark occurs if there is discharge before the gap voltage reaches its nominal level (this level is the gap voltage of normal spark, being about $80 \mathrm{~V}$ in the present case). Consequently, the actual gap voltage of arc sparks has two kinds of time factors. One is the voltage recovery time, which depends on power supply

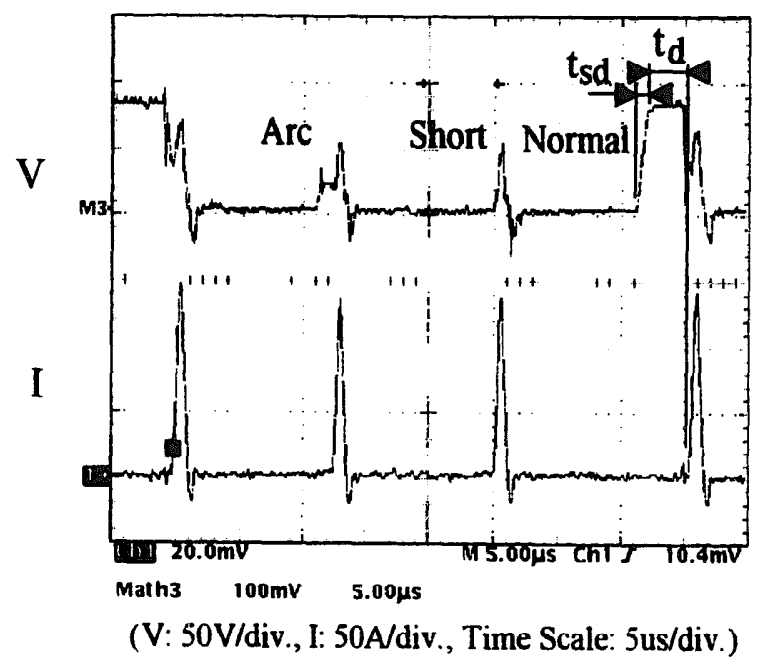

Fig. 3. Typical gap voltage and current waveforms of a WEDM process. 


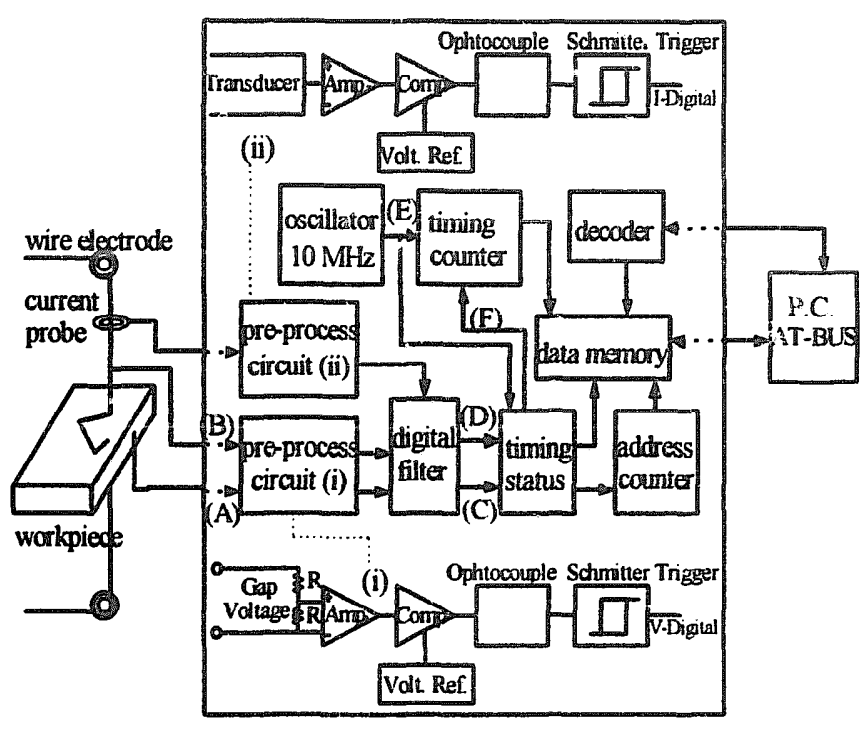

(a)

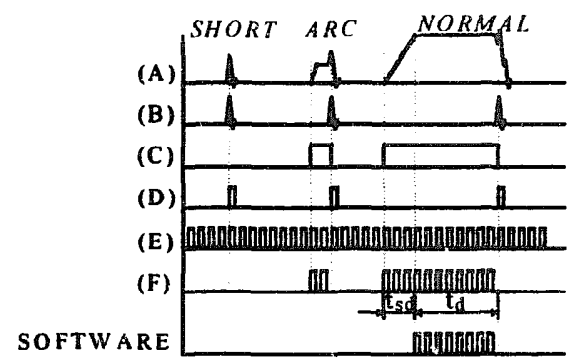

(b)

Fig. 4. The pulse discriminating system of WEDM: (a) system structure: (b) timing chart of the pulse discriminating signal.

capacity and switching ability, and the other is de-ionized time. The latter is influenced by the resistance of the dielectric liquid, which in turn is affected by the gap width and the instantaneous amount of slags in the gap. It is difficult to discriminate arc spark from normal spark because their features are often close. Nevertheless, normal sparks occur when the de-ionized time is longer than the voltage recovery time. This feature is proposed as a discrimination characteristic between are and normal sparks.

A general block diagram of the developed WEDM pulse discriminating system is represented in Fig. 4(a). The gap voltage and the associated current signals of discharge pulses are the input of the system. The current signal is measured by a current probe (Tektronix A6303 type), whilst the voltage signal is obtained by a voltage divider. In the system, two pre-process circuits ((i) and (ii) shown in Fig. 4(a)) and a digital-filter are used to filter and isolate noises resulting from EMI, high-power discharge oscillation and the noise generated by the machine. An address-counter, timingcounter and data-memory cell are supervised by a timing-status urit to record the pulse data converted from analog signals $\mathrm{An}$ IBM $486 \mathrm{PC}$ is used, and communicates with the datid-memory cell through a decoder cell. The pulse trains are analyzed by software written in turbo $\mathrm{C}$ language. Based on this architecture, the pulse data can be recorded and analyzed by the PC simultaneously, hence no successive records are lost. Each sample contains the ignition delay times, with a resolution of $0.1 \mu \mathrm{s}$, of 16384 successive pulses. The timing chart of the puise discriminating signals is shown in Fig. 4(b), in which figures $\mathrm{A}$ and $\mathrm{B}$ are the voltage and the associated current of the sparks, and $C$ and $D$ are the filtering signals of the voltage and current signals, respectively. $E$ is the timing clock signal and $F$ is the time between the instant where the gap voltage starts to rise and the onset of the current of a pulse. The notation $t_{\mathrm{d}}$ is the ignition delay time, whilst the notation $t_{\mathrm{sd}}$ is the threshold level of the time reauired for the gap voltage to rise to its nominal valı.e, the latter depending on the characteristics of the bower supply capacity of the pulse generator. The pulse type can be distinguished according to the duration of the period $F$. The pulse is taken as a short, arc or normal spark by soltware, if the period detected is zero, between zero and $t_{\mathrm{sd}}$, or greater than $t_{\mathrm{sd}}$. The value $t_{\mathrm{sd}}$ can be determined rapidly by an oscilloscope, in the present case it is $1.7 \mu \mathrm{s}$. This kind of discriminating system can be applied to all machining settings.

\section{Experiments}

Experiments are carried out on a prototype iso-energy pulse generator WEDM machine developed by the Mechanical Industrial Resears , Laboratory (MIRL) of ITRI, Taiwan. The curreit has a triangle shape wave-form with a slope of $380 \mathrm{~A} \mu \mathrm{s}^{-1}$. All the tests are conducted under the conditions of a flush rate of 6 bar (both upper and lower guides), a wire tension of $1200 \mathrm{gf}$, and a wire feed of $9 \mathrm{~m} \mathrm{~min}^{-1}$. The work material is SKD 11 tool steel of $50 \mathrm{~mm}$ thickness. The wire electrode is copper with a 0.25 $\mathrm{mm}$ diameter, and a cathodic tool electrode is used. During the experiments, the servo voltage control loop is turned off. A summary of the machining conditions used in the experiments is given in Table 1 , in $t_{\mathrm{o}}$ and $t_{\mathrm{c}}$ are off-time and on-time, respectively, and $f_{\mathrm{d}}$ is the table feed.

In the study, 12 sets of data (each containing 16384 successive pulses) are recorded and analyzed. The ratio of the occurrence of different pulse types, the distribution of the relative frequency of the ignition delay time of normal sparks, the average ignition delay time and the machining time ratio $\left(t_{\mathrm{a}} / t_{\mathrm{t}}\right)$ are evaluated. 
Table 1

Machining conditions used in the experiments

\begin{tabular}{lll}
\hline$t_{\mathrm{o}}(\mu \mathrm{s})$ & $\boldsymbol{t}_{\mathrm{e}}(\mu \mathrm{s})$ & $f_{\mathrm{d}}\left(\mathrm{mm} \mathrm{min}^{-1}\right)$ \\
\hline 11.2 & 0.7 & 2 \\
14.4 & 0.7 & 2 \\
17.6 & 0.7 & 2 \\
20.8 & 0.7 & 2 \\
8 & 0.5 & 2.6 \\
8 & 0.7 & 2.6 \\
8 & 0.9 & 2.6 \\
8 & 1.1 & 2.6 \\
8 & 0.5 & 1.8 \\
8 & 0.5 & 2.0 \\
8 & 0.5 & 2.2 \\
8 & 0.5 & 2.4 \\
\hline
\end{tabular}

\section{Method of estimating the variation of average gap width}

The dynamic gap between the electrode and workpiece is difficult to estimate because of the wire vibration and deflection resulting from the explosive force, the electrostatic force, the electromagnetic force and the flushing force acting on the wire. A method which can be used to estimate the variation of average gap width is illustrated and shown in Fig. 5(a) and 5(b). The wire locations when the gap voltage is applied and the instances of gap breakdown are indicated by $P_{1}$ and $P_{2}$, respectively.

The sparking frequency can be written as:

$F_{\mathrm{q}}=N_{\mathrm{t}} / t_{\mathrm{t}}$

where $N_{\mathrm{t}}$ is the total number of pulses and $t_{\mathrm{t}}$ is the total machining time.

The period of the spark is the reciprocal of $F_{\mathrm{q}}$, i.e.

(a)

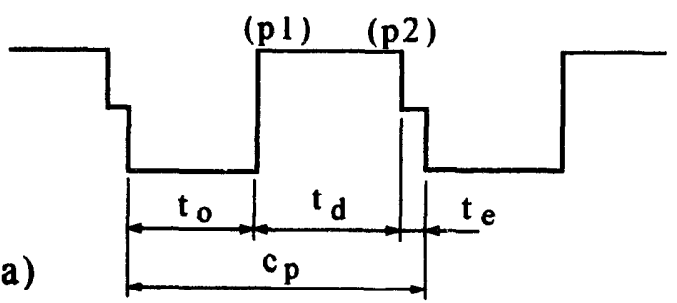

(b)

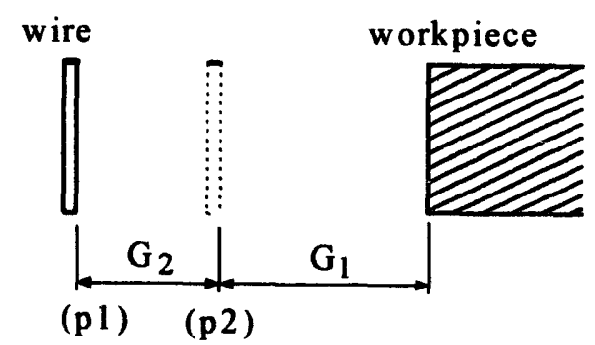

Fig. 5. (a) Showing the period of pulse and (b) the relative location between the wire and the workpiece.

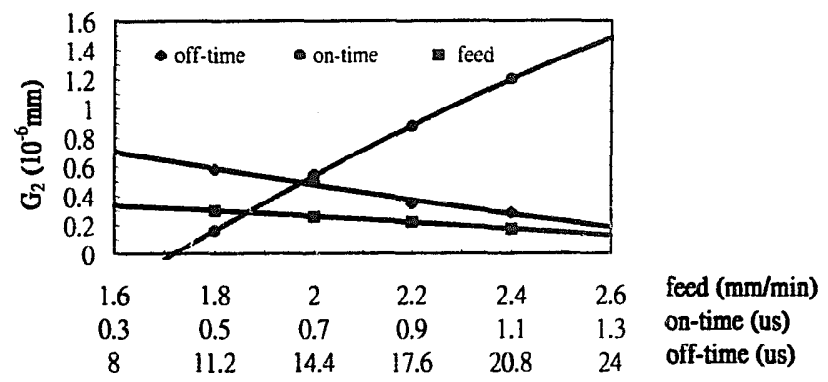

Fig. 6. The effects of machining conditions on $\mathrm{G}_{2}$.

$C_{\mathrm{p}}=1 / F_{\mathrm{q}}$

The value of average ignition delay time during sampling time is then:

$\bar{t}_{\mathrm{D}}=C_{\mathrm{p}}-t_{\mathrm{o}}-t_{\mathrm{e}}$

Hence, the change of the average distance between the wire and the workpiece during $\bar{t}_{\mathrm{D}}$ can be computed as:

$G_{2}=f_{\mathrm{d}} * \bar{t}_{\mathrm{D}}$

The gap width at the breakdown of insulation in a sampling time interval denoted by $G_{1}$ is considered to be equal because the voltage supplied to the gap is constant $(110 \mathrm{~V})$, and the high power of 220 voltage is turned on causing the gap to discharge when the gap voltage is less than the pre-set value $(40 \mathrm{~V})$. If the change of the average distance between the wire and the workpiece within the time of gap voltage $(110 \mathrm{~V})$ rise and breakdown is represented by $G_{2}$, then the variation of electron-gap width depends on $\mathbf{G}_{2}$.

\section{Results and discussion}

The effect of machining conditions on $G_{2}$ is shown in Fig. 6. A short off-time, a long on-time, or a small table feed results in the increase of $G_{2}$, which means that the gap width is increased, and there is a larger breakdown time. The effects of off-time on $R_{\mathrm{n}}, R_{\mathrm{a}}, R_{\mathrm{s}}$ and $\bar{t}_{\mathrm{p}}$ are shown in Fig. 7. An increase of off-time results in a

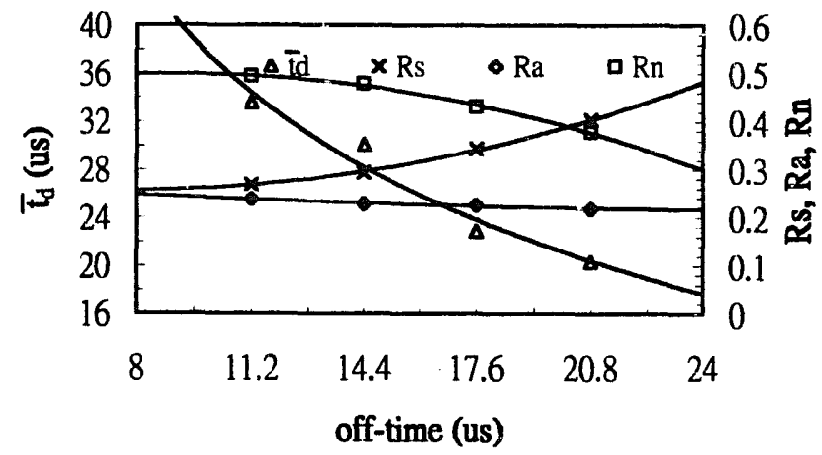

Fig. 7. The effects of off-time on $R_{\mathrm{s}}, R_{\mathrm{a}}, R_{\mathrm{n}}$ and $\bar{t}_{\mathrm{D}}$. 


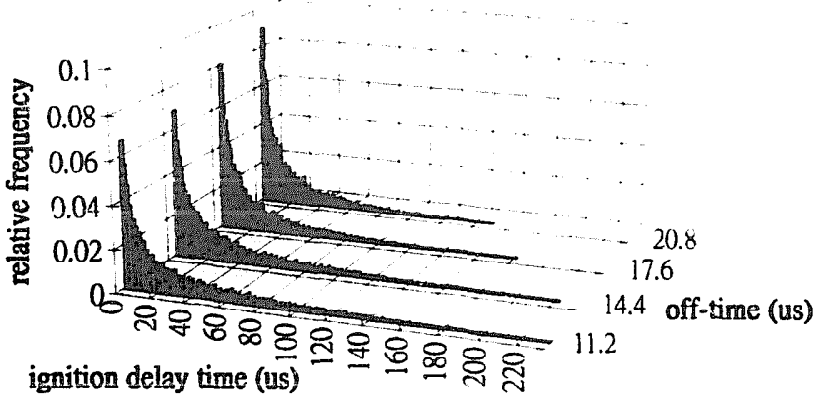

Fig. 8. The effects of off-time on the distribution of the ignition delay time of normal sparks.

reduction of normal ratio and an increase of short ratio. However, the arc ratio is affected insignificantly, the increment being less than $2 \%$. The increase of short ratio is due to the decrease of gap width with the increase of off-time under constant-feed machining conditions. Arcs occur due to early ionization, which depends on the instantaneous concentration of the slag in the electron-gap. The slag volume produced by each discharge pulse is considered to be the same (due to constant on-time). The instantaneous concentration of electron-gap is affected by the gap width and flushing ability. A larger off-time results in a small gap width, but it also offers a longer flushing time to clean the debris in the gap. Hence, there is no significant variation on arc-ratio with the change of off-time. These results seem to contradict common practice, since a longer off-time is always adopted for wire rupture prevention or for the elimination of the abnormal process. The discrepancy is due to the servo voltage control loop which is always implemented in a commercially available machine. It is noted that the increase of off-time leads to the decrease of gap voltage. Hence, the feed-rate is reduced, and the gap width becomes larger accordingly. This action allows a better flush of the debris in the gap, and the process is improved. The relative frequency distribution of ignition delay with respect to off-time is shown in Fig. 8, in which the ignition delay durations are grouped in $1 \mu \mathrm{s}$ intervals. The decrease of off-time makes the left-skew distributions have a lower peak and a longer tail, which means that the occurrence of normal sparks with longer ignition delay is increased. This is reasonable, since the decrease of off-time leads to a larger electron-gap.

The effects of on-time on $R_{\mathrm{n}}, R_{\mathrm{a}}, R_{\mathrm{s}}$ and $\overline{t_{\mathrm{D}}}$ are shown in Fig. 9, which reveal that the increase of the on-time settings result in a longer average ignition delay time, an increase of normal ratio, a decrease of short ratio, and a decreasing and then about $10 \%$ increasing trend of arc ratio. As the on-time is increased, the power supplied to the gap is greater, and more metal is instantaneously removed. The larger electron-gap caused by the increase of on-time (Fig. 6) would de-

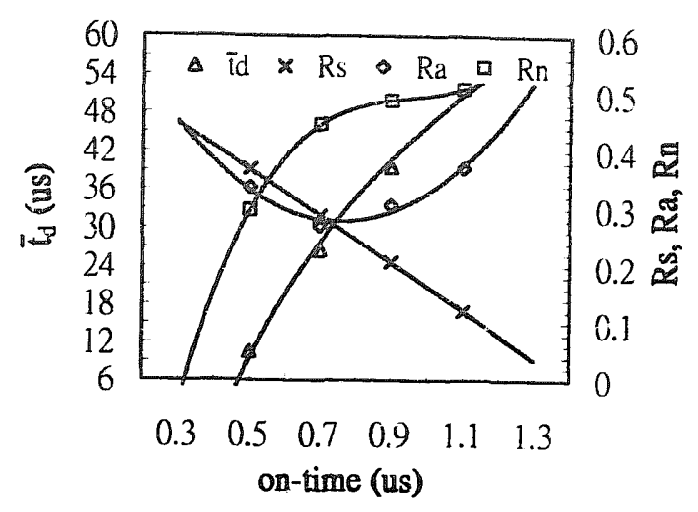

Fig. 9. The effects of on-time on $R_{s}, R_{\mathrm{i}}, R_{\mathrm{n}}$ and $\bar{t}_{\mathrm{D}}$.

crease the probability of metal contact (short ratio is decreased), and result in a longer breakdown time, hence the normal ratio is increased. A larger on-time, such as a $1.1 \mu$ s setting, produces more instantaneous metal removal, which leads to a high instantaneous slag concentration, and the gap becomes casy to ionize. therefore a high arc ratio is seen. For small on-time settings, e.g. $0.5 \mu \mathrm{s}$, there is not much instantaneous metal removal, but a high instantaneous slag concentration still exists because of a small electron-gap, hence, the arc ratio is large also. Since there is a longer ignition delay time with respect to the increase of on-time, the distribution of the ignition delay time of normal sparks will have a lower peak and a longer tail (Fig. 10).

The effects of table feed on $R_{\mathrm{n}}, R_{\mathrm{i}}, R_{\mathrm{s}}$ and $\overline{i_{\mathrm{p}}}$ are shown in Fig. 11, which reveals that an increase of table feed setting results in a decrease of normal ratio, a decrease of average ignition delay time, an increase of short ratio, and a slight increase of arc ratio (less than $4 \%$. Hence, table feed can be used to control both short and arc ratio simultaneously. The deciease of table feed also causes the distribution of the ignition delay time of normal sparks to shift more to the right. Again, a lower peak with a long tail is noticed (Fig. 12). The above variations can be explained similarly by the conditions of gap width and slag concentration.

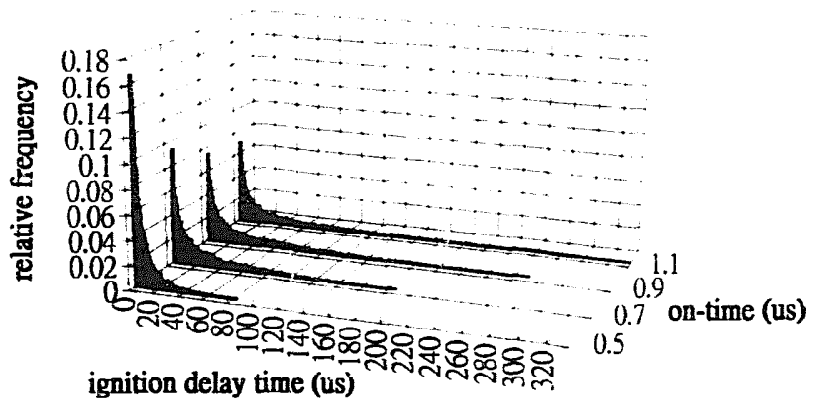

Fig. 10. The effects of on-time on the distribution of ignition delay time of normal sparks. 


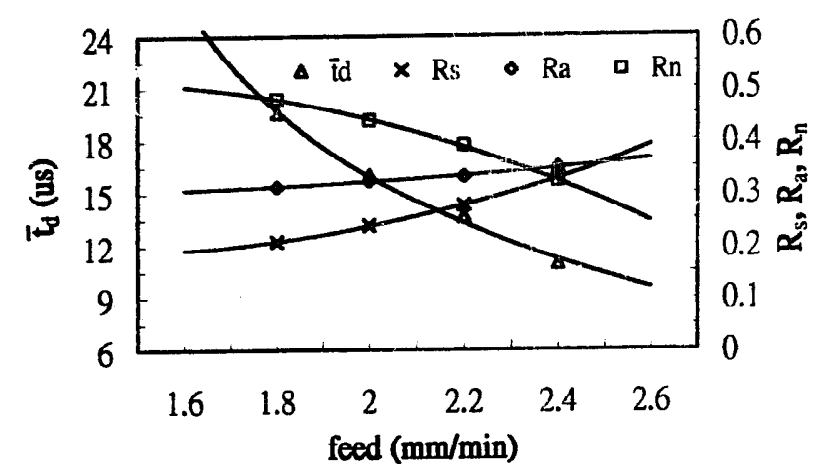

Fig. 11. The effects of table feed on $R_{\mathrm{s}}, R_{\mathrm{a}}, R_{\mathrm{n}}$ and $\bar{t}_{\mathrm{D}}$.

The effect of machining conditions on $t_{\mathrm{a}} / t_{\mathrm{t}}$ is shown in Fig. 13. This quantity can be considered as a rough index of the machining efficiency [2]. Total actual machining time $t_{\mathrm{a}}$ is taken as the summation of actual discharge durations of normal and arc sparks. The notation $t_{\mathrm{t}}$ is the total machining time. There exists a proportional relationship between table feed and machining time ratio, and a reverse relationship between off-time and machining time ratio. The machining time ratio shows a decreasing and then increasing trend with respect to on-time.

\section{Conclusions}

A WEDM pulse discrimination system has been developed which provides a means for more precise online quantitative pulse trains analysis and for machining condition monitoring. A method for estimating the variation of average gap width is proposed. The settings of short on-time, long off-time and high table feed cause the gap to become smaller, which in turn, results in the decrease of normal ratio and average ignition delay time, and an increase of short ratio. On-time has the largest effect, whilst off-time has the least effect on the arc ratio. The ignition delay of normal sparks exhibited a left skew reverse J-shape distribution, this

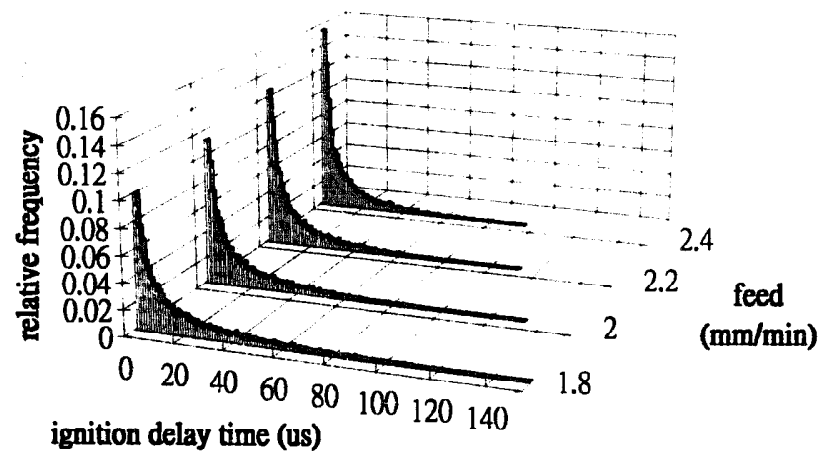

Fig. 12. The effects of table feed on the distribution of ignition delay time of normal sparks.

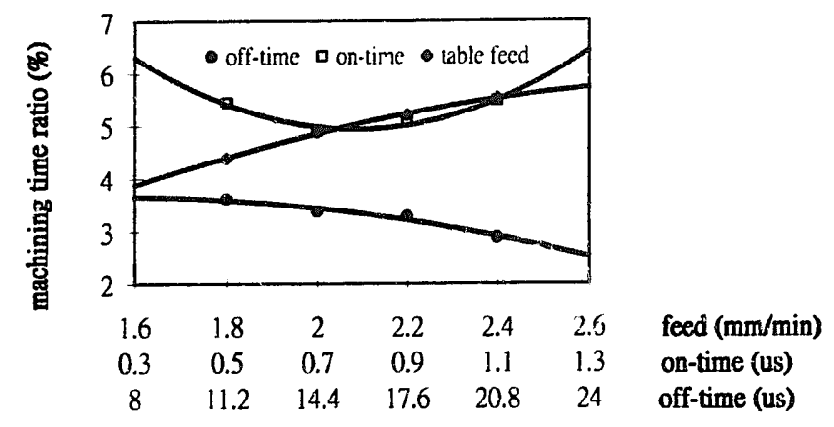

Fig. 13. The effects of machining conditiond on $t_{\mathrm{a}} / t_{\mathrm{t}}$.

distribution being influenced most by on-time. The increase of abnormal sparks due to inappropriate maciniung scitiiigs would result in three kinds of machining instability, namely, the increase of arc, short, and both arc and short sparks. The strategy to improve machining stability is to lower on-time for the first case, whilst off-time is reduced for the second case. However, if there is a high risk of wire rupture, off-time should be increased and table feed should be reduced rather than lower off-time. The table feed should be reduced for the last case. The implementation of the proposed control strategy is in progress.

\section{Acknowledgements}

Support from Ching Hung Machinery and Electric Industrial Co., Ltd., Taiwan is gratefully acknowledged.

\section{References}

[1] J.R. Crookall, A basic analysis of trains in electro-discharge machining, Int. J. Mach. Tool Des. Res. 13 (1972) 199-213.

[2] R. Snoeys, H. Cornelissen, Correlation between electro discharge machining data and machining settings, Ann. CIRP 24 (1975) 83-88.

[3] D.F. Dauw, R. Snoeys, W. Dekeyser, Advanced pulse discriminating system for EDM process analysis and control, Ann. CIRP 32 (2) (1983) $541-549$.

[4] H. Watanabe, T. Stato, I. Suzuki, WEDM monitoring with a statistical pulse classification method, Ann. CIRP 39 (1) (1990) 175-178.

[5] V.S.R. Murthy, P.K. Philip, Pulse train analysis in ultrasonic assisted EDM, Int. J. Mach. Tools Manuf. 27 (4) (1987) 469-477.

[6] C. Coğun, Variation of discharge profile with discharge power in electric discharge machining (EDM), JSME, Int. J. Series 332 (3) (1989) 480-483.

[7] C. Coğun, M. Savsar, Statistical modeling of properties discharge pulses in electric discharge machining, Int. J. Mach. Tools Manuf. 30 (3) (1990) 467-474.

[8] A. Gangadhar, M.S. Shunmugam, P.K. Philip, A pulse train studies in EDM with controlled pulse relaxation, Int. J. Mach. Tools Manuf. 32 (5) (1992) 651-657. 
[9] K.P. Rajurkar, W.M. Wang. WEDM identification and adaptive control for variable-height components, Ann. CIRP 43 (1) (1994) 199-202.

[10] Y.S. Liau, Y.Y. Chu, M.T. Yan, Study of wire Brsaking Process and Monitoring of WEDM. Sino-German Joint Symp. Precision and High Speed Manufacturing Technology. Titipei. Taiman. 1993. pp. $323-338$.

[11] M.T. Yan. Y.S. Liao. Adaptive Control of WEDM Process Using the Fuzzy Control Strategy. Proc. Ilth litl. Symp. Electromachining. iSEM-11. 1995. pp. 34.3 .354$. 